\title{
A dichotomy for bounded displacement equivalence of Delone sets
}

\author{
YOTAM SMILANSKY $\dagger$ and YAAR SOLOMON@ $†$ \\ $\dagger$ Department of Mathematics, Rutgers University, New Brunswick, NJ, USA \\ (e-mail: yotam.smilansky@rutgers.edu) \\ $\$$ Department of Mathematics, Ben-Gurion University of the Negev, Beersheba, Israel \\ (e-mail:yaars@bgu.ac.il)
}

(Received 10 November 2020 and accepted in revised form 4 May 2021)

\begin{abstract}
We prove that in every compact space of Delone sets in $\mathbb{R}^{d}$, which is minimal with respect to the action by translations, either all Delone sets are uniformly spread or continuously many distinct bounded displacement equivalence classes are represented, none of which contains a lattice. The implied limits are taken with respect to the Chabauty-Fell topology, which is the natural topology on the space of closed subsets of $\mathbb{R}^{d}$. This topology coincides with the standard local topology in the finite local complexity setting, and it follows that the dichotomy holds for all minimal spaces of Delone sets associated with well-studied constructions such as cut-and-project sets and substitution tilings, whether or not finite local complexity is assumed.
\end{abstract}

Key words: bounded displacement equivalence, Delone sets, minimal spaces, aperiodic order

2020 Mathematics Subject Classification: 37B05, 37B52, 52C23, 52C25 (Primary)

\section{Introduction}

A set $\Lambda \subset \mathbb{R}^{d}$ is called a Delone set if it is both uniformly discrete and relatively dense, that is, if there are constants $r, R>0$ so that every ball of radius $r$ contains at most one point of $\Lambda$ and $\Lambda$ intersects every ball of radius $R$. We refer to $r$ and $R$ as the separation constant and the packing radius of $\Lambda$, respectively. Two Delone sets $\Lambda, \Gamma \subset \mathbb{R}^{d}$ are said to be bounded displacement (BD)-equivalent if there exists a bijection $\phi: \Lambda \rightarrow \Gamma$ satisfying

$$
\sup _{\mathbf{x} \in \Lambda}\|\mathbf{x}-\phi(\mathbf{x})\|<\infty .
$$

Such a mapping $\phi$ is called a $B D$-map. Note that because norms in $\mathbb{R}^{d}$ are equivalent, this definition does not depend on the choice of norm. Lattices in $\mathbb{R}^{d}$ with the same covolume are BD-equivalent, and a Delone set $\Lambda$ is called uniformly spread if it is equivalent to a lattice or, equivalently, if there is a BD-map $\phi: \Lambda \rightarrow \alpha \mathbb{Z}^{d}$, for some $\alpha>0$. 
By fixing a metric $\rho$ on $\mathbb{R}^{d}$ and considering the space $\mathscr{C}\left(\mathbb{R}^{d}\right)$ of closed subsets of $\mathbb{R}^{d}$, the Chabauty-Fell topology on $\mathscr{C}\left(\mathbb{R}^{d}\right)$ is the topology induced by the metric (see Appendix A)

$$
\left.D\left(\Lambda_{0}, \Lambda_{1}\right) \stackrel{\text { def }}{=} \inf \left(\begin{array}{l|l}
\varepsilon>0 & \begin{array}{l}
\Lambda_{0} \cap B(\mathbf{0}, 1 / \varepsilon) \subset \Lambda_{1}^{(+\varepsilon)} \\
\Lambda_{1} \cap B(\mathbf{0}, 1 / \varepsilon) \subset \Lambda_{0}^{(+\varepsilon)}
\end{array}
\end{array}\right\} \cup\{1\}\right),
$$

where $B(\mathbf{x}, R)$ is the open ball of radius $R>0$ centered at $\mathbf{x} \in \mathbb{R}^{d}$ with respect to the metric $\rho$, and $A^{(+\varepsilon)}$ is the $\varepsilon$ neighborhood of the set $A$. In this work, we only consider metrics $\rho$ that are determined by norms on $\mathbb{R}^{d}$, and although different choices of norms result in different metrics $D$, they all define the same topology. We remark that in the aperiodic order literature, this topology, which was introduced by Chabauty [Ch] for $\mathscr{C}\left(\mathbb{R}^{d}\right)$ as well as for a more general setting, and later extended by Fell [Fe], is often referred to as the natural topology or the local rubber topology, see e.g. [BG, §5]. See also [LSt] for the relation to the Hausdorff metric.

Delone sets in $\mathbb{R}^{d}$ are elements of $\mathscr{C}\left(\mathbb{R}^{d}\right)$, and we may consider compact spaces of Delone sets, where the implied limits are taken with respect to the Chabauty-Fell topology. Such a space $\mathbb{X}$ of Delone sets in $\mathbb{R}^{d}$ is minimal with respect to the $\mathbb{R}^{d}$ action by translations if the orbit closure of every Delone set $\Lambda \in \mathbb{X}$ is dense in $\mathbb{X}$. Minimality of $\mathbb{X}$ is equivalent to the recurrence of patches in each Delone set $\Lambda \in \mathbb{X}$, where a patch is a finite subset of a Delone set. This important geometric consequence of minimality is called almost repetitivity, and a precise definition and additional details are given in $\S 3$. For a proof of this equivalence, see [FR, Theorem 3.11] and [SS, Theorem 6.5], and see also the discussion included in $[\mathrm{KL}]$.

By denoting the cardinality of the set of BD-equivalence classes represented in $\mathbb{X}$ by $\mathrm{BD}(\mathbb{X})$, the following dichotomy is our main result.

THEOREM 1.1. Let $\mathbb{X}$ be a space of Delone sets in $\mathbb{R}^{d}$, and assume it is compact with respect to the Chabauty-Fell topology and minimal with respect to the action of $\mathbb{R}^{d}$ by translations. Then either:

(1) there exists a uniformly spread Delone set in $\mathbb{X}$ (and so every $\Lambda \in \mathbb{X}$ is uniformly spread and $\mathrm{BD}(\mathbb{X})=1)$; or

(2) $\mathrm{BD}(\mathbb{X})=2^{\aleph_{0}}$;

where $2^{\aleph_{0}}$ denotes the cardinality of the continuum.

Observe that the minimality assumption is essential, as shown by the following simple example. Consider $\Lambda=(-2 \mathbb{N}) \sqcup\{0\} \sqcup \mathbb{N}$, a Delone set in $\mathbb{R}$. Then the orbit closure $\mathbb{X}$ of $\Lambda$ under translations by $\mathbb{R}$ and with respect to the Chabauty-Fell topology consists of translations of $\Lambda$, the orbit closure of $\mathbb{Z}$ and the orbit closure of $2 \mathbb{Z}$. Therefore, $\operatorname{BD}(\mathbb{X})=3$ and, indeed, $\mathbb{X}$ is not minimal.

Let us describe the proof of Theorem 1.1. The implication in the brackets of (1) is a direct consequence of [La, Theorem 1.1], see also [FG, Theorem 3.2] for a sketch of a similar proof that holds for general minimal spaces of Delone sets. A uniformly discrete set in $\mathbb{R}^{d}$ with separation constant $r>0$ is BD-equivalent to a subset of the lattice $(r / 2) \mathbb{Z}^{d}$, hence the upper bound $\mathrm{BD}(\mathbb{X}) \leq 2^{\aleph_{0}}$ is trivial. We prove the remaining implication according to the following steps. Given a non-uniformly spread Delone set 
in a minimal space $\mathbb{X}$, we construct in $\S 4$ a sequence of pairs of patches consisting of an increasingly deviant number of points. In $\S 3$, choosing a patch from each pair, which corresponds to the choice of a word on a two letter alphabet, gives rise to a Delone sets in $\mathbb{X}$ with certain properties. Finally, it is shown in $\S 5$ that by using the equivalent condition for non-BD equivalence of two Delone sets established in $\$ 2$, two Delone sets defined using words that differ in infinitely many places are BD-non-equivalent, and so $\mathbb{X}$ contains continuously many BD-equivalence classes.

Recall that a Delone set $\Lambda$ has finite local complexity (FLC) if, for every $R>0$, the number of distinct patterns that are contained in balls of radius $R$ in $\Lambda$ up to translations is finite. In such a case, every Delone set in the orbit closure of $\Lambda$ under translations, sometimes called the hull of $\Lambda$, also has FLC. The hull itself is then called FLC, and the Chabauty-Fell topology on $\mathbb{X}$ coincides with the local topology, see [BG, §5]. It follows that Theorem 1.1 holds also for FLC spaces with respect to the local topology, and constitutes a new result both in the FLC and non-FLC set-up. In particular, it answers question (1) in [FG, §7] in the strongest possible way.

In addition to Theorem 1.1, in Theorem 2.3, we establish a useful equivalent condition for two Delone sets to be non-BD equivalent. This result is the converse of the implication of Theorem 2.2, which first appeared in [FSS], and may be of interest in its own right.

Delone sets are mathematical models of atomic positions, and BD-equivalence offers a natural way of classifying them. BD-equivalence for general discrete point sets was previously considered mainly in the context of uniformly spread point sets, see e.g. [D01, D02, DSS, La]. In recent years, BD-equivalence has emerged as an object of study for Delone sets that appear in the study of mathematical quasicrystals and aperiodic order, see $[\mathrm{BG}]$ for a comprehensive introduction to such constructions. For cut-and-project sets, BD-equivalence was studied in [HKW], and links to the notions of bounded remainder sets and pattern equivariant cohomology appeared in [FG, HK, HKK] and in [KS1, KS2], respectively. For Delone sets associated with substitution tilings, sufficient conditions for a set to be uniformly spread were provided in [ACG, S1, S2]. In addition, for the multiscale substitution tilings introduced by the authors in [SS], it was shown that any Delone set associated with an incommensurable tiling cannot be uniformly spread.

Recently, questions regarding BD-non-equivalence between two Delone sets were considered in [FSS], where a sufficient condition for BD-non-equivalence was established. It was later shown in [S3] that if the eigenvalues and eigenspaces of the substitution matrix satisfy a certain condition, then the corresponding substitution tiling space contains continuously many distinct BD-classes.

The following less-restrictive equivalence relation on Delone sets is often studied in parallel to the BD-equivalence relation. We say that two Delone sets $\Lambda$ and $\Gamma$ are biLipschitz (BL)-equivalent if there exists a BL bijection between them. Namely, a bijection $\varphi: \Lambda \rightarrow \Gamma$ and a constant $C \geq 1$ so that

$$
\text { for all } \mathbf{x}, \mathbf{y} \in \Lambda, \quad \frac{1}{C} \leq \frac{\|\varphi(\mathbf{x})-\varphi(\mathbf{y})\|}{\|\mathbf{x}-\mathbf{y}\|} \leq C .
$$

It was shown by Burago and Kleiner [BK1], and independently by McMullen [McM], Delone sets exist in $\mathbb{R}^{d}, d \geq 2$, that are not BL-equivalent to a lattice in $\mathbb{R}^{d}$. It was shown in 
[Mag] that there are continuously many Delone sets that are pairwise BL-non-equivalent, and a hierarchy of equivalence relations on Delone sets, which includes BD and BL equivalence, was recently introduced in [DK]. It would be interesting to obtain an analogue of our Theorem 1.1 in this context.

Question 1.2. Does Theorem 1.1 hold if BD-equivalence is replaced by BL-equivalence?

In view of the sufficient condition for BL-equivalence to a lattice, given by Burago and Kleiner in [BK2] and the constructions in [CN, Mag], we remark that the results given in $\S \S 3$ and 4 , with respect to densities and discrepancy estimates, may be relevant also in the study of BL-non-equivalence and the question stated above.

1.1. Consequences of Theorem 1.1. Theorem 1.1 directly implies that $\mathrm{BD}(\mathbb{X})=2^{\aleph_{0}}$ for many special families of minimal spaces of Delone sets, which are central in the theory of aperiodic order and for which the BD-equivalence relation was considered previously.

1.1.1. Substitution tilings. For primitive substitution tilings of $\mathbb{R}^{d}$, we denote the eigenvalues of the substitution matrix by $\lambda_{1}>\left|\lambda_{2}\right| \geq \cdots \geq\left|\lambda_{n}\right|$, and we let $t \geq 2$ be the minimal index such that the eigenspace of $\lambda_{t}$ contains non-zero vectors whose sum of coordinates is not zero. Under the assumption that tiles are BL homeomorphic to closed balls, it was shown in [S2, Theorem 1.2 (I)] that if

$$
\left|\lambda_{t}\right|>\lambda_{1}^{(d-1) / d}
$$

then the Delone sets corresponding to the tilings in the tiling space are not uniformly spread. Under the assumption (1.2) and an additional assumption regarding the existence of certain patches, it was recently shown in $[\mathrm{S3}]$ that $\mathrm{BD}(\mathbb{X})=2^{\aleph_{0}}$. Given the above result of [S2], and because substitution tiling spaces are minimal (see [BG]), the following strengthening of the main result of [S3] is a direct consequence of our Theorem 1.1.

COROLLARY 1.3. Let $\mathbb{X}$ be a primitive substitution tiling space with tilings by tiles that are $B L$ homeomorphic to closed balls. Assume that condition (1.2) holds, then $\mathrm{BD}(\mathbb{X})=2^{\aleph_{0}}$.

Note that in the context of tilings, we say that two tilings are BD-equivalent if their corresponding Delone sets, which are obtained by picking a point from each tile, are BD-equivalent. In addition to the above, [S2] contains an example of a substitution rule, for which the eigenvalues of the substitution matrix satisfy

$$
\left|\lambda_{2}\right|=\lambda_{1}^{(d-1) / d}
$$

and the corresponding Delone sets are not uniformly spread, see [S2, Theorem 1.2 (III)]. Note that in this example, the main result of [S3] cannot be applied.

COROLlARY 1.4. There exists a primitive substitution tiling space $\mathbb{X}$ for which condition (1.3) holds and $\mathrm{BD}(\mathbb{X})=2^{\aleph_{0}}$.

1.1.2. Cut-and-project sets. Theorem 1.2 in [HKW] concerns the BD-equivalence relation in the context of cut-and-project sets that arise from linear toral flows (which constitute 
an equivalent method of constructing cut-and-project sets, see [ASW, Proposition 2.3]). Because the hull of a cut-and-project set is minimal, the next corollary follows directly from [HKW, Theorem 1.2 (III)] and our Theorem 1.1. We refer to [HKW] for more details on the construction and terminology.

COROLlary 1.5. For almost every $(k-d)$-dimensional linear section $S$, which is a parallelotope in the $k$-dimensional torus, there is a residual set of d-dimensional subspaces $V$ for which the hull of the corresponding cut-and-project set contains continuously many distinct BD-classes.

The half-Fibonacci sets were introduced in [FG, §6]. These are cut-and-project sets in $\mathbb{R}$ that belong to the same hull and are BD-non-equivalent. In particular, they are not uniformly spread (see [FG, Theorem 3.2]). We thus obtain the following result.

COROllary 1.6. Let $\mathbb{X}$ be the hull of the half-Fibonacci sets from [FG]. Then $\mathrm{BD}(\mathbb{X})=2^{\aleph_{0}}$.

1.1.3. Multiscale substitution tilings. Multiscale substitution tilings were studied recently in [SS]. Under an incommensurability assumption on the underlying substitution scheme, the corresponding tiling spaces are minimal [SS, §6], and combined with a mild assumption on the boundaries of the prototiles, which holds for example for polygonal tiles, their associated Delone sets, which are never FLC, are also never uniformly spread [SS, §8].

COROLlary 1.7. Let $\mathbb{X}$ be an incommensurable multiscale polygonal tiling space. Then $\mathrm{BD}(\mathbb{X})=2^{\aleph_{0}}$.

A proof of Theorem 1.1 in the FLC set-up was given in [FGS], which appeared after the first version of this paper came out. Their work is independent of ours.

\section{Necessary and sufficient conditions for BD-non-equivalence}

2.1. Notations. Bold characters are used to denote vectors in $\mathbb{R}^{d}$, and we use the supremum norm $\|\cdot\|_{\infty}$ on $\mathbb{R}^{d}$ throughout this paper. Note that with respect to this norm, balls are (Euclidean) cubes, and we use both terms interchangeably. We denote by $\partial A,|A|$ and $\operatorname{vol}(A)$ the boundary, cardinality and Lebesgue measure of a set $A \subset \mathbb{R}^{d}$, respectively, and we denote the cardinality of a finite set $S$ by $\# S$. Given $\varepsilon>0$ and $A \subset \mathbb{R}^{d}$, we denote the $\varepsilon$-neighborhood of $A$ by

$$
A^{(+\varepsilon)} \stackrel{\text { def }}{=}\left\{\mathbf{x} \in \mathbb{R}^{d} \mid \operatorname{dist}(\mathbf{x}, A) \leq \varepsilon\right\},
$$

where $\operatorname{dist}(\mathbf{x}, A)=\inf \left\{\|\mathbf{x}-\mathbf{a}\|_{\infty} \mid \mathbf{a} \in A\right\}$. For an integer $m>0$, we denote the collection of all half-open cubes in $\mathbb{R}^{d}$ with edge-length $m$ and with vertices in $m \mathbb{Z}^{d}$ by

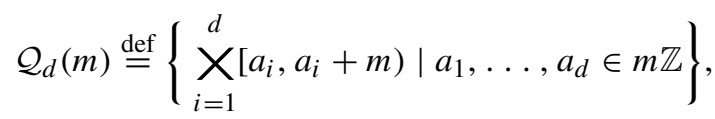


and we denote the collection of finite unions of elements from $\mathcal{Q}_{d}(m)$ by $\mathcal{Q}_{d}^{*}(m)$. In the case $m=1$, we simply write $\mathcal{Q}_{d}$ and $\mathcal{Q}_{d}^{*}$. For $A \in \mathcal{Q}_{d}$, the term $\operatorname{vol}_{d-1}(\partial A)$ denotes the $(d-1)$-Lebesgue measure of $\partial A$. The following lemma is a direct consequence of Lemmas 2.1 and 2.2 of [La].

Lemma 2.1. Let $F$ be a translated copy of an element of $\mathcal{Q}_{d}^{*}$ and let $s>0$, then

$$
\operatorname{vol}\left((\partial F)^{(+s)}\right) \leq c_{0} \cdot s^{d} \cdot \operatorname{vol}_{d-1}(\partial F),
$$

where $c_{0}$ depends only on $d$.

2.2. BD-equivalence. The following condition for non-BD-equivalence of two Delone sets in $\mathbb{R}^{d}$ was given in [FSS].

THEOREM 2.2. [FSS, Theorem 1.1] Let $\Lambda_{0}, \Lambda_{1}$ be two Delone sets in $\mathbb{R}^{d}$ and suppose that there is a sequence $\left(A_{m}\right)_{m \in \mathbb{N}}$ of sets, $A_{m} \in \mathcal{Q}_{d}^{*}$, for which

$$
\frac{\left|\#\left(\Lambda_{0} \cap A_{m}\right)-\#\left(\Lambda_{1} \cap A_{m}\right)\right|}{\operatorname{vol}_{d-1}\left(\partial A_{m}\right)} \stackrel{m \rightarrow \infty}{\longrightarrow} \infty .
$$

Then there is no BD-map $\phi: \Lambda_{0} \rightarrow \Lambda_{1}$.

We show that the converse also holds (compare with [La, Lemma 2.3]).

THEOREM 2.3. The following are equivalent for two Delone sets $\Lambda_{0}, \Lambda_{1}$ in $\mathbb{R}^{d}$.

(i) There is no BD-map between $\Lambda_{0}$ and $\Lambda_{1}$.

(ii) There is a sequence $\left(A_{m}\right)_{m \in \mathbb{N}}$ of sets, which are translated copies of elements of $\mathcal{Q}_{d}^{*}$, such that

$$
\frac{\left|\#\left(\Lambda_{0} \cap A_{m}\right)-\#\left(\Lambda_{1} \cap A_{m}\right)\right|}{\operatorname{vol}_{d-1}\left(\partial A_{m}\right)} \stackrel{m \rightarrow \infty}{\longrightarrow} \infty .
$$

Proof. The implication (ii) $\Rightarrow$ (i) follows from Theorem 2.2, because translating the sets $A_{m}$ by at most $\sqrt{d}$ changes the numerator by, at most, a constant times $\operatorname{vol}_{d-1}\left(\partial A_{m}\right)$.

For (i) $\Rightarrow$ (ii), suppose that there is no BD-map between $\Lambda_{0}$ and $\Lambda_{1}$, that is, no bijection $\phi: \Lambda_{0} \rightarrow \Lambda_{1}$ that satisfies

$$
\sup _{\mathbf{x} \in \Lambda_{0}}\|\mathbf{x}-\phi(\mathbf{x})\|_{\infty}<\infty
$$

For every $m \in \mathbb{N}$, consider the bipartite graph $\mathcal{G}_{m} \stackrel{\text { def }}{=}\left(\Lambda_{0} \sqcup \Lambda_{1}, \mathcal{E}_{m}\right)$, where

$$
\mathcal{E}_{m}=\left\{\{\mathbf{x}, \mathbf{y}\} \mid \mathbf{x} \in \Lambda_{0}, \mathbf{y} \in \Lambda_{1},\|\mathbf{x}-\mathbf{y}\|_{\infty} \leq 2 m\right\}
$$

The existence of a perfect matching in $\mathcal{G}_{m}$ for some $m$ would imply the existence of a BD-map between $\Lambda_{0}$ and $\Lambda_{1}$, which contradicts our assumption. Thus, by Hall's marriage theorem (see e.g. [Ra]), for every $m \in \mathbb{N}$, there is a set $X_{m} \subset \Lambda_{i_{m}}, i_{m} \in\{0,1\}$, so that $\# X_{m}>\#\left(X_{m}^{(+2 m)} \cap \Lambda_{1-i_{m}}\right)$. By fixing $m \in \mathbb{N}$ and assuming, without loss of generality, that $i_{m}=0$, set

$$
A_{m} \stackrel{\text { def }}{=} \bigcup\left\{Q \in \mathcal{Q}_{d}(m) \mid Q \cap X_{m} \neq \varnothing\right\} \in \mathcal{Q}_{d}^{*}(m) .
$$


For $Q \in \mathcal{Q}_{d}(m)$, let $Q^{\prime}$ be a cube of edge-length $3 m$, which is concentric with $Q$, and set

$$
B_{m} \stackrel{\text { def }}{=} \bigcup\left\{Q^{\prime} \mid Q \in \mathcal{Q}_{d}(m), Q \cap X_{m} \neq \varnothing\right\} \in \mathcal{Q}_{d}^{*}(m) .
$$

Clearly, $B_{m} \supset A_{m} \supset X_{m}$ and, by the triangle inequality, we have $X_{m}^{(+2 m)} \supset B_{m}$. Therefore,

$$
\#\left(\Lambda_{0} \cap A_{m}\right)>\#\left(\Lambda_{1} \cap B_{m}\right)=\#\left(\Lambda_{1} \cap A_{m}\right)+\#\left(\Lambda_{1} \cap\left(B_{m} \backslash A_{m}\right)\right),
$$

which implies

$$
\#\left(\Lambda_{0} \cap A_{m}\right)-\#\left(\Lambda_{1} \cap A_{m}\right)>\#\left(\Lambda_{1} \cap\left(B_{m} \backslash A_{m}\right)\right) .
$$

It is left to show that $\#\left(\Lambda_{1} \cap\left(B_{m} \backslash A_{m}\right)\right) / \operatorname{vol}_{d-1}\left(\partial A_{m}\right) \stackrel{m \rightarrow \infty}{\longrightarrow} \infty$, which is a consequence of the following argument taken from the proof of [La, Lemma 2.3]. Suppose that $\partial A_{m}$ consists of $s$ faces of cubes in $\mathcal{Q}_{d}(m)$. For each such face, let $P_{j}$ be the cube in $\mathcal{Q}_{d}(m)$ contained in $B_{m} \backslash A_{m}$ with a boundary containing that face. Note that $P_{1}, \ldots, P_{s}$ are not necessarily distinct and that each cube has $2 d$ faces, and so

$$
2 d \cdot \operatorname{vol}\left(B_{m} \backslash A_{m}\right) \geq \sum_{j=1}^{s} \operatorname{vol}\left(P_{j}\right)=s \cdot m^{d}=m \cdot s \cdot m^{d-1}=m \cdot \operatorname{vol}_{d-1}\left(\partial A_{m}\right) .
$$

The relative denseness of $\Lambda_{1}$ implies that $\#\left(\Lambda_{1} \cap\left(B_{m} \backslash A_{m}\right)\right) \geq c \cdot \operatorname{vol}\left(B_{m} \backslash A_{m}\right)$ for some constant $c>0$ independent of $m$, and the proof follows.

COROLlary 2.4. Let $\left(A_{m}\right)_{m \in \mathbb{N}}$ be a sequence of sets as in (2.2), then for every $R>0$, there exists $M>0$ so that for every $m \geq M$, each $A_{m}$ contains a ball of radius $R$.

Proof. Let $R>0$ and suppose that there is a sequence $m_{j} \rightarrow \infty$ such that for every $j$, the set $A_{m_{j}}$ does not contain a ball of radius $R$. Then for every $j$, we have $A_{m_{j}} \subset\left(\partial A_{m_{j}}\right)^{(+R)}$ and thus, by Lemma 2.1,

$$
\operatorname{vol}\left(A_{m_{j}}\right) \leq c_{0} \cdot R^{d} \cdot \operatorname{vol}_{d-1}\left(\partial A_{m_{j}}\right) .
$$

Because $\Lambda_{0}$ and $\Lambda_{1}$ are uniformly discrete and relatively dense, there exist constants $a, b>$ 0 so that for every $j$,

$$
a \cdot \operatorname{vol}\left(A_{m_{j}}\right) \leq \#\left(\Lambda_{0} \cap A_{m_{j}}\right), \#\left(\Lambda_{1} \cap A_{m_{j}}\right) \leq b \cdot \operatorname{vol}\left(A_{m_{j}}\right) .
$$

Combining the above implies that for every $j$, we have

$$
\frac{\left|\#\left(\Lambda_{0} \cap A_{m_{j}}\right)-\#\left(\Lambda_{1} \cap A_{m_{j}}\right)\right|}{\operatorname{vol}_{d-1}\left(\partial A_{m_{j}}\right)} \leq(b-a) c_{0} \cdot R^{d},
$$

which contradicts (2.2).

\section{The topology on spaces of Delone sets}

We consider the dynamical system $(X, d, G)$, where $(X, d)$ is a compact metric space and $G$ is a group acting on $X$. The dynamical system $(X, d, G)$ is called minimal if every $G$-orbit, $G . x \stackrel{\text { def }}{=}\{g . x \mid g \in G\}$ for $x \in X$, is dense in $(X, d)$. A set $S \subset G$ is called syndetic if there is a compact set $K \subset G$ so that for every $g \in G$, there is a $k \in K$ with $k g \in S$. Note 
that when $G=\mathbb{R}^{d}$, this notion coincides with our definition of a relatively dense set. A point $x_{0} \in X$ is said to be uniformly recurrent if, for every open neighborhood $U$ of $x_{0}$, the set of 'return times' to $U,\left\{g \in G \mid g . x_{0} \in U\right\}$, is syndetic. As shown in [Fu, Theorem 1.15], in minimal systems, every point is uniformly recurrent.

Recall that given a metric $\rho$ on $\mathbb{R}^{d}$, we may use (1.1) to define a metric $D$ on $\mathscr{C}\left(\mathbb{R}^{d}\right)$, the space of closed subsets of $\left(\mathbb{R}^{d}, \rho\right)$, and that this metric induces the Chabauty-Fell topology. Here and in what follows, we take $\rho$ to be the metric defined by the supremum norm $\|\cdot\|_{\infty}$ on $\mathbb{R}^{d}$. Note that replacing it with any other norm on $\mathbb{R}^{d}$, such as the Euclidean norm, would change the metric $D$ but not the induced topology, also known as the local rubber topology in the context of aperiodic order. It is known that $D$ is a complete metric on $\mathscr{C}\left(\mathbb{R}^{d}\right)$, and the space $\left(\mathscr{C}\left(\mathbb{R}^{d}\right), D\right)$ is compact, see e.g. [dH, LSt].

Let $\mathbb{X}$ be a collection of Delone sets in $\mathbb{R}^{d}$. Under the additional assumptions, where $\mathbb{X}$ is a closed subset of $\mathscr{C}\left(\mathbb{R}^{d}\right)$ and that $\mathbb{R}^{d}$ acts on $\mathbb{X}$ by translations, the space $\left(\mathbb{X}, D, \mathbb{R}^{d}\right)$ is a compact dynamical system. We say that $\Lambda \in \mathbb{X}$ is almost repetitive if, for every $\mathbf{x} \in \mathbb{R}^{d}$ and $\varepsilon>0$, there exists $R=R(\varepsilon, \mathbf{x})>0$ such that every ball $B(\mathbf{y}, R)$ in $\mathbb{R}^{d}$ contains a vector $\mathbf{v} \in \mathbb{R}^{d}$ that satisfies

$$
D(\Lambda-\mathbf{x}, \Lambda-\mathbf{v})<\varepsilon .
$$

In words, for every $\mathbf{x} \in \mathbb{R}^{d}$ and $\varepsilon>0$, there exists $R>0$ so that a copy of $B(\mathbf{0}, 1 / \varepsilon) \cap$ $(\Lambda-\mathbf{x})$ can be found in every $R$-ball, up to wiggling each point by at most $\varepsilon$. We also refer to [FR, Definitions 2.8, 2.13, and 3.5] and to [LP] for distinctions between similar definitions of repetitivity.

The observation in Lemma 3.1 is useful when working with the metric $D$ in spaces of uniformly discrete point sets.

LEMMA 3.1. Suppose that $\Lambda_{0}, \Lambda_{1} \subset \mathbb{R}^{d}$ are uniformly discrete sets with separation constant $r>0$, and that $D\left(\Lambda_{0}, \Lambda_{1}\right)<\varepsilon$ for $0<\varepsilon<r / 2$. Then, for every set $A \subset$ $B(0,1 / \varepsilon)$ that is a translated copy of an element of $\mathcal{Q}_{d}^{*}$, there exist injective maps

$$
\varphi_{0}: \Lambda_{0} \cap A \rightarrow \Lambda_{1} \cap A^{(+\varepsilon)}, \quad \varphi_{1}: \Lambda_{1} \cap A \rightarrow \Lambda_{0} \cap A^{(+\varepsilon)},
$$

that satisfy

for all $\mathbf{x} \in \Lambda_{0} \cap A, \quad\left\|\mathbf{x}-\varphi_{0}(\mathbf{x})\right\|_{\infty}<\varepsilon, \quad$ for all $\mathbf{y} \in \Lambda_{1} \cap A, \quad\left\|\mathbf{y}-\varphi_{1}(\mathbf{y})\right\|_{\infty}<\varepsilon$.

In particular, there is a constant $c_{1}$ that depends on $d$ and $r$ so that

$$
\left|\#\left(\Lambda_{0} \cap A\right)-\#\left(\Lambda_{1} \cap A\right)\right| \leq c_{1} \cdot \varepsilon^{d} \cdot \operatorname{vol}_{d-1}(\partial A) .
$$

Proof. Given $A \subset B(\mathbf{0}, 1 / \varepsilon)$ as above, because $D\left(\Lambda_{0}, \Lambda_{1}\right)<\varepsilon$, the existence of $\varphi_{0}, \varphi_{1}$ satisfying (3.1) follows directly from the definition of $D$ in (1.1). Note that the maps are injective because $\varepsilon<r / 2$. Therefore,

$$
\left|\#\left(\Lambda_{0} \cap A\right)-\#\left(\Lambda_{1} \cap A\right)\right| \leq \#\left(\Lambda_{0} \cap(\partial A)^{(+\varepsilon)}\right)+\#\left(\Lambda_{1} \cap(\partial A)^{(+\varepsilon)}\right) .
$$

Because $\Lambda_{0}$ and $\Lambda_{1}$ are uniformly discrete, and in view of Lemma 2.1, (3.2) follows. 
We remark that if $\Lambda$ is a Delone set in $\mathbb{R}^{d}$ with separation constant and packing radius $r, R>0$, and if $\mathbb{X}$ is the orbit closure of $\Lambda$ with respect to $D$, then every $\Gamma \in \mathbb{X}$ is a Delone set with separation constant at least $r$ and packing radius at most $R$.

The following lemma shows that minimal spaces are uniformly almost repetitive. Namely, the radius $R(\mathbf{x}, \varepsilon)$, from the definition of almost repetitivity above, does not depend on $\mathbf{x}$.

LEMMA 3.2. Let $\mathbb{X}$ be a compact space of Delone sets so that the dynamical system $\left(\mathbb{X}, D, \mathbb{R}^{d}\right)$ is minimal. Then, for every $0<\varepsilon<1$, there exists $R=R(\varepsilon)>0$, so that for every $\Lambda, \Gamma \in \mathbb{X}$ and $\mathbf{y} \in \mathbb{R}^{d}$, there exists some $\mathbf{v} \in B(\mathbf{y}, R)$ for which

$$
D(\Gamma, \Lambda-\mathbf{v})<\varepsilon .
$$

Proof. Let $\varepsilon>0$, and let $\Lambda \in \mathbb{X}$ and $\mathbf{x} \in \mathbb{R}^{d}$. By minimality, the set $\Lambda-\mathbf{x}$ is uniformly recurrent. For $\eta>0$, denote $U_{\eta}^{\mathbf{x}} \stackrel{\text { def }}{=}\left\{\Lambda^{\prime} \in \mathbb{X} \mid D\left(\Lambda-\mathbf{x}, \Lambda^{\prime}\right)<\eta\right\}$, then the set $\left\{\mathbf{v} \in \mathbb{R}^{d} \mid\right.$ $\left.\Lambda-\mathbf{v} \in U_{\varepsilon / 2}^{\mathbf{x}}\right\}$ is relatively dense (syndetic). Therefore, there exists $R_{\varepsilon / 2}^{\mathbf{x}}>0$ such that every cube of edge-length $R_{\varepsilon / 2}^{\mathbf{x}}$ in $\mathbb{R}^{d}$ contains some $\mathbf{v} \in \mathbb{R}^{d}$ satisfying $D(\Lambda-\mathbf{x}, \Lambda-$ v) $<\varepsilon / 2$.

By minimality again, the collection $\left\{\Lambda-\mathbf{x} \mid \mathbf{x} \in \mathbb{R}^{d}\right\}$ is dense in $\mathbb{X}$. Thus, $\left\{U_{\varepsilon / 2}^{\mathbf{x}}\right\}_{x \in \mathbb{R}^{d}}$ is an open cover of $\mathbb{X}$ and, by compactness, there exists a finite sub-cover $U_{\varepsilon / 2}^{\mathbf{x}_{1}}, \ldots, U_{\varepsilon / 2}^{\mathbf{x}_{n}}$. Then, for every $\Gamma \in \mathbb{X}$, there exists some $j \in\{1, \ldots, n\}$ so that $\Gamma \in U_{\varepsilon / 2}^{\mathbf{x}_{j}}$, and hence $D\left(\Gamma, \Lambda-\mathbf{x}_{j}\right)<\varepsilon / 2$. Setting $R \stackrel{\text { def }}{=} \max \left\{R_{\varepsilon / 2}^{\mathbf{x}_{1}}, \ldots, R_{\varepsilon / 2}^{\mathbf{x}_{n}}\right\}$, it follows that for every $\mathbf{y} \in \mathbb{R}^{d}$, there exists some $\mathbf{v} \in B\left(\mathbf{y}, R_{\varepsilon / 2}^{\mathbf{x}_{j}}\right) \subset B(\mathbf{y}, R)$ such that $D\left(\Lambda-\mathbf{x}_{j}, \Lambda-\mathbf{v}\right)<\varepsilon / 2$. Then, by the triangle inequality, $D(\Gamma, \Lambda-\mathbf{v})<\varepsilon$, as required.

In Proposition 3.3 below, we consider a Delone set $\Lambda$ in a minimal space, and show that if sets $A_{m}$ in $\mathcal{Q}_{d}^{*}$ grow sufficiently fast, then there exist translation vectors $\mathbf{u}_{m}$ so that the patches $Q_{m}=\left(\Lambda \cap A_{m}\right)-\mathbf{u}_{m}$ converge to a limit object that 'almost' contains all of the patches. The idea of the proof is simply to use the almost repetitivity property to inductively find an 'almost' copy of $Q_{m-1}$ inside $\Lambda \cap A_{m}$, and to set $\mathbf{u}_{m}$ so that it is centered accordingly, namely, so that the copy we find 'almost' agrees with $Q_{m-1}$. Note that every sequence of sets that grows in a reasonable sense has a subsequence that grows fast enough to satisfy conditions (1) and (2) in Proposition 3.3.

Proposition 3.3. Let $\mathbb{X}$ be a minimal space of Delone sets in $\mathbb{R}^{d}, \Lambda \in \mathbb{X},\left(A_{m}\right)_{m \in \mathbb{N}}$ a sequence of sets in $\mathcal{Q}_{d}^{*}$ and $\left(\varepsilon_{m}\right)_{m \geq 0}$ a decreasing sequence of positive constants with $\varepsilon_{0}<\min \{1, r(\Lambda) / 2\}$, where $r(\Lambda)$ is the separation constant of $\Lambda$. For every $m \geq 0$, choose $R\left(\varepsilon_{m}\right)$ satisfying Lemma 3.2 and set $\left.R_{m} \stackrel{\text { def }}{=} \max \left\{R\left(\varepsilon_{m}\right), 1 / \varepsilon_{m}\right)\right\}$. Assume that the following properties hold for every $m \in \mathbb{N}$ :

(1) there exists $\mathbf{x}_{m} \in \mathbb{R}^{d}$ such that $A_{m} \subset B\left(\mathbf{x}_{m}, 1 / 2 \varepsilon_{m}\right)$;

(2) there exists $\mathbf{y}_{m} \in \mathbb{R}^{d}$ such that $B\left(\mathbf{y}_{m}, 2 R_{m-1}\right) \subset A_{m}$. 
Then there exist $\mathbf{u}_{m} \in B\left(\mathbf{y}_{m}, R_{m-1}\right)$ and patches $Q_{m} \stackrel{\text { def }}{=}\left(\Lambda \cap A_{m}\right)-\mathbf{u}_{m}$ such that $\lim _{m \rightarrow \infty} Q_{m}=\Gamma \in \overline{\mathbb{R}^{d} . \Lambda}=\mathbb{X}$. Moreover, for every $m \geq 2$,

$$
\begin{gathered}
B\left(\boldsymbol{0}, R_{m-1}\right) \subset A_{m}-\mathbf{u}_{m} \subset B\left(\boldsymbol{0}, 1 / \varepsilon_{m}\right), \\
D\left(\Lambda-\mathbf{u}_{m-1}, \Lambda-\mathbf{u}_{m}\right)<\varepsilon_{m-1}, \\
D\left(Q_{m}, \Gamma\right)<\varepsilon_{m-1}
\end{gathered}
$$

and there exists $c_{2}>0$ so that

$$
\left|\#\left(\Gamma \cap\left(A_{m}-\mathbf{u}_{m}\right)\right)-\# Q_{m}\right| \leq c_{2} \cdot \varepsilon_{m}^{d} \cdot \operatorname{vol}_{d-1}\left(\partial A_{m}\right),
$$

where $c_{2}$ depends on the dimension $d$ and separation constant $r(\Lambda)$.

Proof. First, observe that by assumptions (1) and (2),

$$
\varepsilon_{m+1} \leq \frac{1}{4 R_{m}} \leq \frac{1}{4} \varepsilon_{m}
$$

holds for every $m \in \mathbb{N}$. In particular, the series $\sum_{m=1}^{\infty} \varepsilon_{m}$ is convergent.

We define the vectors $\mathbf{u}_{m}$, and hence the patches $Q_{m}$, inductively.

- By (1), $A_{1}$ is in particular contained in a ball of radius $1 / \varepsilon_{1}$. Let $\mathbf{u}_{1}$ be such that $Q_{1}=\left(\Lambda \cap A_{1}\right)-\mathbf{u}_{1}$ is contained in $B\left(\mathbf{0}, 1 / \varepsilon_{1}\right)$.

Assume that the vectors $\mathbf{u}_{j}$, and thus the patches $Q_{j}=\left(\Lambda \cap A_{j}\right)-\mathbf{u}_{j}$, are defined for $j \in\{1, \ldots, m\}$ such that for every $2 \leq j \leq m$, we have

(i) $B\left(\mathbf{0}, R_{j-1}\right) \subset A_{j}-\mathbf{u}_{j} \subset B\left(\mathbf{0}, 1 / \varepsilon_{j}\right)$.

(ii) $D\left(\Lambda-\mathbf{u}_{j}, \Lambda-\mathbf{u}_{j-1}\right)<\varepsilon_{j-1}$.

We define $\mathbf{u}_{m+1}$ as follows.

- By (2), $A_{m+1}$ contains a ball of the form $B\left(\mathbf{y}_{m+1}, 2 R_{m}\right)$. By Lemma 3.2, let $\mathbf{u}_{m+1} \in$ $B\left(\mathbf{y}_{m+1}, R_{m}\right)$ be a vector satisfying

$$
D\left(\Lambda-\mathbf{u}_{m}, \Lambda-\mathbf{u}_{m+1}\right)<\varepsilon_{m} .
$$

Thus (ii) holds for $j=m+1$. Note that because $B\left(\mathbf{y}_{m+1}, 2 R_{m}\right) \subset A_{m+1}$ and $\mathbf{u}_{m+1} \in$ $B\left(\mathbf{y}_{m+1}, R_{m}\right)$, we have

$$
B\left(\mathbf{0}, R_{m}\right) \subset A_{m+1}-\mathbf{u}_{m+1} .
$$

By (1), $A_{m+1}-\mathbf{u}_{m+1} \subset B\left(\mathbf{x}_{m+1}-\mathbf{u}_{m+1}, 1 / 2 \varepsilon_{m+1}\right)$ and so $A_{m+1}-\mathbf{u}_{m+1}$ contains the origin. Then, by the triangle inequality, $A_{m+1}-\mathbf{u}_{m+1}$ is contained in $B\left(\mathbf{0}, 1 / \varepsilon_{m+1}\right)$, which completes the proof of (i) for $j=m+1$.

This completes the construction of the vectors $\mathbf{u}_{m}$ and the patches $Q_{m}$. Next, we show that the sequence $\left(Q_{m}\right)_{m \in \mathbb{N}}$ is a Cauchy sequence. By fixing some $\varepsilon>0$ and letting $M$ be so that $2 \varepsilon_{M}<\varepsilon$, we let $m>n>M$ and note that by property (ii), we have $D$ ( $\Lambda$ $\left.\mathbf{u}_{k+1}, \Lambda-\mathbf{u}_{k}\right)<\varepsilon_{k}$, for every $k \geq M$. Then, by the triangle inequality,

$$
D\left(\Lambda-\mathbf{u}_{m}, \Lambda-\mathbf{u}_{n}\right) \leq \sum_{k=n}^{m-1} D\left(\Lambda-\mathbf{u}_{k+1}, \Lambda-\mathbf{u}_{k}\right)<\sum_{k=n}^{m-1} \varepsilon_{k}<2 \varepsilon_{n}<2 \varepsilon_{M}<\varepsilon,
$$


where the third inequality follows from (3.7). By property (i), for every $j \in \mathbb{N}$, the point sets $Q_{j}$ and $\Lambda-\mathbf{u}_{j}$ in particular coincide on the ball $B\left(\mathbf{0}, 1 / \varepsilon_{j-1}\right)$. Because $m, n>M$, the sets $\Lambda-\mathbf{u}_{n}$ and $Q_{n}$ coincide on $B(\mathbf{0}, 1 / \varepsilon)$, and similarly for $\Lambda-\mathbf{u}_{m}$ and $Q_{m}$. Therefore, relying on (3.8), for every $m>n>M$, we have

$$
D\left(Q_{m}, Q_{n}\right) \leq D\left(\left(\Lambda-\mathbf{u}_{m}\right) \cap B(\mathbf{0}, 1 / \varepsilon),\left(\Lambda-\mathbf{u}_{n}\right) \cap B(\mathbf{0}, 1 / \varepsilon)\right)<\varepsilon .
$$

Thus, $\left(Q_{m}\right)_{m \in \mathbb{N}}$ is a Cauchy sequence. The space $(\mathbb{X}, D)$ is complete, as a compact metric space, hence the limit $\Gamma \stackrel{\text { def }}{=} \lim _{m \rightarrow \infty} Q_{m}=\lim _{m \rightarrow \infty} \Lambda-\mathbf{u}_{m}$ exists and belongs to $\mathbb{X}$.

It is left to prove (3.3), (3.4), (3.5), and (3.6). First, observe that (3.3) and (3.4) follow immediately from the construction, see properties (i) and (ii). To see (3.5), let $m \in \mathbb{N}$ and let $k>m$ be so that $D\left(Q_{k}, \Gamma\right)<\varepsilon_{m}$. Repeating the computations in (3.8) and (3.9) yields that $D\left(Q_{m}, Q_{k}\right)<2 \varepsilon_{m}$, and by (3.7), we have

$$
D\left(Q_{m}, \Gamma\right) \leq D\left(Q_{m}, Q_{k}\right)+D\left(Q_{k}, \Gamma\right)<3 \varepsilon_{m}<\varepsilon_{m-1} .
$$

Finally, we prove (3.6). By (3.3), we have $A_{m}-\mathbf{u}_{m} \subset B\left(\mathbf{0}, 1 / \varepsilon_{m}\right)$ and by (3.5), we have $D\left(\Gamma, Q_{m+1}\right)<\varepsilon_{m}$. Thus, by Lemma 3.1 with $A=A_{m}-\mathbf{u}_{m}$, we obtain

$$
\left|\#\left(\Gamma \cap\left(A_{m}-\mathbf{u}_{m}\right)\right)-\#\left(Q_{m+1} \cap\left(A_{m}-\mathbf{u}_{m}\right)\right)\right| \leq c_{1} \cdot \varepsilon_{m}^{d} \cdot \operatorname{vol}_{d-1}\left(\partial A_{m}\right) .
$$

By (3.4), we have $D\left(\Lambda-\mathbf{u}_{m}, \Lambda-\mathbf{u}_{m+1}\right)<\varepsilon_{m}$, and applying Lemma 3.1 once again, we obtain

$$
\left|\#\left(\left(\Lambda-\mathbf{u}_{m+1}\right) \cap\left(A_{m}-\mathbf{u}_{m}\right)\right)-\#\left(\left(\Lambda-\mathbf{u}_{m}\right) \cap\left(A_{m}-\mathbf{u}_{m}\right)\right)\right| \leq c_{1} \cdot \varepsilon_{m}^{d} \cdot \operatorname{vol}_{d-1}\left(\partial A_{m}\right) .
$$

By the definition of $Q_{m}$, and because $A_{m}-\mathbf{u}_{m} \subset A_{m+1}-\mathbf{u}_{m+1}$ by (3.3), this is exactly

$$
\left|\#\left(Q_{m+1} \cap\left(A_{m}-\mathbf{u}_{m}\right)\right)-\# Q_{m}\right| \leq c_{1} \cdot \varepsilon_{m}^{d} \cdot \operatorname{vol}_{d-1}\left(\partial A_{m}\right) .
$$

Combining this with (3.10) yields (3.6) and completes the proof of the theorem.

\section{Finding patches with large discrepancy}

The goal of this section is to prove the following proposition, which will be used in our proof of Theorem 1.1 in $\S 5$.

Proposition 4.1. Let $\Lambda \subset \mathbb{R}^{d}$ be a non-uniformly spread Delone set. Then there exist a sequence $\left(A_{m}\right)_{m \in \mathbb{N}}$ of sets in $\mathcal{Q}_{d}^{*}$ and a sequence $\left(\mathbf{x}_{m}\right)_{m \in \mathbb{N}}$ of vectors in $\mathbb{Z}^{d}$ so that

$$
\frac{\left|\#\left(\Lambda \cap A_{m}\right)-\#\left(\Lambda \cap\left(A_{m}+\mathbf{x}_{m}\right)\right)\right|}{\operatorname{vol}_{d-1}\left(\partial A_{m}\right)} \stackrel{m \rightarrow \infty}{\longrightarrow} \infty .
$$

Let $\Lambda \subset \mathbb{R}^{d}$ be a Delone set. We define the central lower density and the central upper density of $\Lambda$ respectively by

$$
\Delta_{*}(\Lambda) \stackrel{\text { def }}{=} \liminf _{t \rightarrow \infty} \frac{\#(B(\mathbf{0}, t) \cap \Lambda)}{\operatorname{vol}(B(\mathbf{0}, t))}, \quad \Delta^{*}(\Lambda) \stackrel{\text { def }}{=} \limsup _{t \rightarrow \infty} \frac{\#(B(\mathbf{0}, t) \cap \Lambda)}{\operatorname{vol}(B(\mathbf{0}, t))} .
$$

If the $\operatorname{limit}_{t \rightarrow \infty} \#(B(\mathbf{0}, t) \cap \Lambda) / \operatorname{vol}(B(\mathbf{0}, t))$ exists, it is called the central density of $\Lambda$ and is denoted by $\Delta(\Lambda)$.

We begin with the following lemma. 
Lemma 4.2. Let $\Lambda$ be a Delone set, $\gamma>0$, and $A \in \mathcal{Q}_{d}^{*}$. Then, for every $\varepsilon>0$, there exists $K>0$ such that for every integer $k \geq K$ :

(1) if $\#(\Lambda \cap B(\boldsymbol{0}, k)) / \operatorname{vol}(B(\boldsymbol{0}, k)) \geq \gamma$, then the ball $B(\boldsymbol{0}, k)$ contains $A+\mathbf{x}$, a translated copy of $A$ with $\mathbf{x} \in \mathbb{Z}^{d}$, such that

$$
\frac{\#(\Lambda \cap(A+\mathbf{x}))}{\operatorname{vol}(A)} \geq \gamma-\varepsilon
$$

(2) if $\#(\Lambda \cap B(\boldsymbol{0}, k)) / \operatorname{vol}(B(\boldsymbol{0}, k)) \leq \gamma$, then the ball $B(\boldsymbol{0}, k)$ contains $A+\mathbf{x}$, a translated copy of $A$ with $\mathbf{x} \in \mathbb{Z}^{d}$, such that

$$
\frac{\#(\Lambda \cap(A+\mathbf{x}))}{\operatorname{vol}(A)} \leq \gamma+\varepsilon .
$$

Proof. This is a simple averaging argument. We prove property (1), the proof of property (2) is similar.

By denoting the diameter of the set $A$ by $\rho$, for a large integer $k$, we write $B(\mathbf{0}, k)=$ $B_{[-\rho]} \sqcup(\partial B)_{[+\rho]}$, where $B_{[-\rho]},(\partial B)_{[+\rho]} \in \mathcal{Q}_{d}^{*}$ are defined by

$$
\begin{aligned}
B_{[-\rho]} & \stackrel{\text { def }}{=} \bigcup\left\{Q \in \mathcal{Q}_{d} \mid Q \subset B(\mathbf{0}, k), \operatorname{dist}(Q, \partial B(\mathbf{0}, k))>\rho\right\}, \\
(\partial B)_{[+\rho]} & \stackrel{\text { def }}{=} B(\mathbf{0}, k) \backslash B_{[-\rho]},
\end{aligned}
$$

where $\operatorname{dist}(X, Y) \stackrel{\text { def }}{=} \inf \left\{\|\mathbf{x}-\mathbf{y}\|_{\infty} \mid \mathbf{x} \in X, \mathbf{y} \in Y\right\}$.

Given $\varepsilon>0$, we pick $K \in \mathbb{N}$ large enough so that for every integer $k \geq K$, we have

$$
\frac{\operatorname{vol}\left((\partial B)_{[+\rho]}\right)}{\operatorname{vol}(B(\mathbf{0}, k))}<\frac{\varepsilon}{2} .
$$

Let $k \geq K$, such that

$$
\frac{\#(\Lambda \cap B(\mathbf{0}, k))}{\operatorname{vol}(B(\mathbf{0}, k))} \geq \gamma,
$$

and let $N_{k} \stackrel{\text { def }}{=}\left\{\mathbf{x} \in \mathbb{Z}^{d} \mid A+\mathbf{x} \subset B(\mathbf{0}, k)\right\}$. By way of contradiction, assume that

$$
\text { for all } \mathbf{x} \in N_{k}, \quad \#(\Lambda \cap(A+\mathbf{x}))<(\gamma-\varepsilon) \operatorname{vol}(A) .
$$

Note that the number of cubes from $\mathcal{Q}_{d}$ that form $A$ is $\operatorname{vol}(A)$. Then, by counting the points of $\Lambda$ (with multiplicity) in all the sets $A+\mathbf{x}, \mathbf{x} \in N_{k}$, the points in every unit lattice cube in $B_{[-\rho]}$ is counted exactly $\operatorname{vol}(A)$ times. Thus,

$$
\# N_{k}(\gamma-\varepsilon) \operatorname{vol}(A) \stackrel{(4.5)}{>} \sum_{\mathbf{x} \in N_{k}} \#(\Lambda \cap(A+\mathbf{x})) \geq \operatorname{vol}(A) \cdot \#\left(\Lambda \cap B_{[-\rho]}\right) .
$$

Note that $\# N_{k} \leq \operatorname{vol}(B(\mathbf{0}, k))$, then dividing both sides of $(4.6)$ by $\operatorname{vol}(A) \cdot \operatorname{vol}(B(\mathbf{0}, k))$ yields

$$
\gamma-\varepsilon>\frac{\#\left(\Lambda \cap B_{[-\rho]}\right)}{\operatorname{vol}(B(\mathbf{0}, k))} \stackrel{(4.2)}{\geq} \frac{\#(\Lambda \cap B(\mathbf{0}, k))}{\operatorname{vol}(B(\mathbf{0}, k))}-\frac{\#\left(\Lambda \cap(\partial B)_{[+\rho]}\right)}{\operatorname{vol}(B(\mathbf{0}, k))} \stackrel{(4.3),(4.4)}{>} \gamma-\frac{\varepsilon}{2},
$$

which is a contradiction. 
Lemma 4.3. Suppose that $\Lambda$ is a Delone set in $\mathbb{R}^{d}$ and that $\Delta_{*}(\Lambda)<\Delta^{*}(\Lambda)$. Then there exist $\alpha<\beta$, integers $a_{k} \rightarrow \infty$ and $\mathbf{x}_{k} \in \mathbb{Z}^{d}$, such that

$$
\frac{\#\left(\Lambda \cap B\left(\boldsymbol{0}, a_{k}\right)\right)}{\operatorname{vol}\left(B\left(\boldsymbol{0}, a_{k}\right)\right)} \leq \alpha \quad \text { and } \quad \frac{\#\left(\Lambda \cap B\left(\mathbf{x}_{k}, a_{k}\right)\right)}{\operatorname{vol}\left(B\left(\mathbf{x}_{k}, a_{k}\right)\right)} \geq \beta .
$$

Proof. By the assumption on the densities, there exist sequences $a_{k}, b_{l} \rightarrow \infty$ so that

$$
\lim _{k \rightarrow \infty} \frac{\#\left(\Lambda \cap B\left(\mathbf{0}, a_{k}\right)\right)}{\operatorname{vol}\left(B\left(\mathbf{0}, a_{k}\right)\right)}=\tilde{\alpha} \quad \text { and } \quad \lim _{l \rightarrow \infty} \frac{\#\left(\Lambda \cap B\left(\mathbf{0}, b_{l}\right)\right)}{\operatorname{vol}\left(B\left(\mathbf{0}, b_{l}\right)\right)}=\tilde{\beta},
$$

where $\tilde{\alpha}<\tilde{\beta}$. Because $\Lambda$ is uniformly discrete, and because the $(d-1)$-volume of the boundary of a cube grows slower than the volume of the cube, we may assume that the numbers $a_{k}, b_{k}$ are integers. Let $\delta<(\tilde{\beta}-\tilde{\alpha}) / 3$ and fix $K \in \mathbb{N}$ such that for every $k, l \geq K$, we have

$$
\frac{\#\left(\Lambda \cap B\left(\mathbf{0}, a_{k}\right)\right)}{\operatorname{vol}\left(B\left(\mathbf{0}, a_{k}\right)\right)} \leq \tilde{\alpha}+\delta \quad \text { and } \quad \frac{\#\left(\Lambda \cap B\left(\mathbf{0}, b_{l}\right)\right)}{\operatorname{vol}\left(B\left(\mathbf{0}, b_{l}\right)\right)} \geq \tilde{\beta}-\delta .
$$

For every $k$, applying Lemma 4.2 with $A=B\left(\mathbf{0}, a_{k}\right), \varepsilon=((\tilde{\beta}-\tilde{\alpha}) / 3)-\delta>0$, and $\tilde{\beta}-\delta$ in the role of $\gamma$, and combining this with (4.7), we find a large enough $l=l_{k}$ and $\mathbf{x}_{k} \in \mathbb{Z}^{d}$ so that $B\left(\mathbf{0}, b_{l}\right)$ contains the ball $B\left(\mathbf{x}_{k}, a_{k}\right)$, which satisfies

$$
\frac{\#\left(\Lambda \cap B\left(\mathbf{x}_{k}, a_{k}\right)\right)}{\operatorname{vol}\left(B\left(\mathbf{x}_{k}, a_{k}\right)\right)} \geq(\tilde{\beta}-\delta)-\varepsilon=\tilde{\beta}-\frac{\tilde{\beta}-\tilde{\alpha}}{3} .
$$

Setting $\alpha \stackrel{\text { def }}{=} \tilde{\alpha}+((\tilde{\beta}-\tilde{\alpha}) / 3)$ and $\beta \stackrel{\text { def }}{=} \tilde{\beta}-((\tilde{\beta}-\tilde{\alpha}) / 3)$, the assertion follows from (4.7) and (4.8).

Proof of Proposition 4.1. Let $\Lambda \subset \mathbb{R}^{d}$ be a non-uniformly spread Delone set. In view of Lemma 4.3, we may further assume that $\Delta \stackrel{\text { def }}{=} \Delta(\Lambda)$ exists. For $\alpha \neq \Delta^{-1 / d}$, the Delone sets $\alpha \mathbb{Z}^{d}$ and $\Lambda$ do not have the same central density and, hence, there is no BD-map between them (see e.g. [FSS, Corollary 3.2]). By our assumption on $\Lambda$, there is no BD-map between $\Lambda$ and $\Delta^{-1 / d} \mathbb{Z}^{d}$ as well. Applying Theorem 2.3 on these two Delone sets, we obtain a sequence $\left(A_{m}\right)_{m \in \mathbb{N}}$ of sets in $\mathcal{Q}_{d}^{*}$ that satisfies

$$
\frac{\left|\#\left(\Delta^{-1 / d} \mathbb{Z}^{d} \cap A_{m}\right)-\#\left(\Lambda \cap A_{m}\right)\right|}{\operatorname{vol}_{d-1}\left(\partial A_{m}\right)} \stackrel{m \rightarrow \infty}{\longrightarrow} \infty .
$$

By passing to a subsequence of $\left(A_{m}\right)_{m \in \mathbb{N}}$, we may assume that

$$
\frac{\#\left(\Delta^{-1 / d} \mathbb{Z}^{d} \cap A_{m}\right)-\#\left(\Lambda \cap A_{m}\right)}{\operatorname{vol}_{d-1}\left(\partial A_{m}\right)} \stackrel{m \rightarrow \infty}{\longrightarrow} \infty,
$$

and complete the proof using (1) of Lemma 4.2. In the case where $\#\left(\Delta^{-1 / d} \mathbb{Z}^{d} \cap A_{m}\right)<$ $\#\left(\Lambda \cap A_{m}\right)$ for all large values of $m$, the proof is similar using (2) of Lemma 4.2 instead of (1).

For every $m \in \mathbb{N}$, we pick $\varepsilon_{m}$, such that

$$
\varepsilon_{m} \operatorname{vol}\left(A_{m}\right)<\operatorname{vol}_{d-1}\left(\partial A_{m}\right)
$$

and apply Lemma 4.2 with $\gamma=\Delta-\varepsilon_{m}, A=A_{m}$ and $\varepsilon=\varepsilon_{m}$. Note that because $\Delta(\Lambda)=\Delta$ exists, the condition $\#(\Lambda \cap B(\mathbf{0}, k)) / \operatorname{vol}(B(\mathbf{0}, k)) \geq \Delta-\varepsilon_{m}$ is satisfied for 
any sufficiently large $k$. By (1) of Lemma 4.2, in particular, there exists a vector $\mathbf{x}_{m} \in \mathbb{Z}^{d}$ so that

$$
\frac{\#\left(\Lambda \cap\left(A_{m}+\mathbf{x}_{m}\right)\right)}{\operatorname{vol}\left(A_{m}\right)} \geq \Delta-2 \varepsilon_{m} .
$$

From (4.9),

$$
\begin{aligned}
& \frac{\#\left(\Delta^{-1 / d} \mathbb{Z}^{d} \cap A_{m}\right)-\#\left(\Lambda \cap\left(A_{m}+\mathbf{x}_{m}\right)\right)}{\operatorname{vol}_{d-1}\left(\partial A_{m}\right)} \\
& \quad+\frac{\#\left(\Lambda \cap\left(A_{m}+\mathbf{x}_{m}\right)\right)-\#\left(\Lambda \cap A_{m}\right)}{\operatorname{vol}_{d-1}\left(\partial A_{m}\right)} \stackrel{m \rightarrow \infty}{\longrightarrow} \infty .
\end{aligned}
$$

Note that

$$
\#\left(\Delta^{-1 / d} \mathbb{Z}^{d} \cap A_{m}\right) \leq \Delta \cdot \operatorname{vol}\left(A_{m}\right)+c \cdot \operatorname{vol}_{d-1}\left(\partial A_{m}\right),
$$

where $c$ depends on $d$ and $\Delta$, and from (4.11), we also have

$$
\left(\Lambda \cap\left(A_{m}+\mathbf{x}_{m}\right)\right) \geq\left(\Delta-2 \varepsilon_{m}\right) \operatorname{vol}\left(A_{m}\right) .
$$

Then

$$
\begin{gathered}
\#\left(\Delta^{-1 / d} \mathbb{Z}^{d} \cap A_{m}\right)-\#\left(\Lambda \cap\left(A_{m}+\mathbf{x}_{m}\right)\right) \leq c \cdot \operatorname{vol}_{d-1}\left(\partial A_{m}\right)+2 \varepsilon_{m} \operatorname{vol}\left(A_{m}\right) \\
\stackrel{(4.10)}{\leq} c^{\prime} \cdot \operatorname{vol}_{d-1}\left(\partial A_{m}\right),
\end{gathered}
$$

where $c^{\prime}$ depends on $d$ and $\Delta$. Plugging this into (4.12) completes the proof.

\section{Proof of Theorem 1.1}

Given a non-uniformly spread Delone set $\Lambda \subset \mathbb{R}^{d}$, let $A_{m} \in \mathcal{Q}_{d}^{*}$ and $\mathbf{x}_{m} \in \mathbb{Z}^{d}$ be as in Proposition 4.1. Let $\varepsilon_{m}>0$ be so that $A_{m}$ is contained in a ball of radius $1 / 2 \varepsilon_{m}$. Passing to subsequences, by Corollary 2.4 combined with (4.1), we may assume that $A_{m}$ contains a ball of radius $2 R_{m-1}$, where $R_{m}$ is as in Proposition 3.3. We thus have

$$
B\left(\mathbf{y}_{m}, 2 R_{m-1}\right) \subset A_{m} \subset B\left(\mathbf{z}_{m}, 1 / 2 \varepsilon_{m}\right)
$$

for some $\mathbf{y}_{m}, \mathbf{z}_{m} \in \mathbb{R}^{d}$. By denoting

$$
B_{m} \stackrel{\text { def }}{=} A_{m}+\mathbf{x}_{m}, \quad \mathbf{p}_{m} \stackrel{\text { def }}{=} \mathbf{y}_{m}+\mathbf{x}_{m}, \quad \mathbf{q}_{m} \stackrel{\text { def }}{=} \mathbf{z}_{m}+\mathbf{x}_{m},
$$

then

$$
B\left(\mathbf{p}_{m}, 2 R_{m-1}\right) \subset B_{m} \subset B\left(\mathbf{q}_{m}, 1 / 2 \varepsilon_{m}\right),
$$

and so $\left(A_{m}\right)_{m \in \mathbb{N}}$ and $\left(B_{m}\right)_{m \in \mathbb{N}}$ both satisfy Proposition 3.3.

From (4.1), there is a sequence of constants $\mu_{m} \rightarrow \infty$, such that

$$
\left|\#\left(\Lambda \cap A_{m}\right)-\#\left(\Lambda \cap\left(A_{m}+\mathbf{x}_{m}\right)\right)\right|=\mu_{m} \cdot \operatorname{vol}_{d-1}\left(\partial A_{m}\right) .
$$

Because $\mu_{m} \rightarrow \infty$, by passing to a further subsequence, we may assume that $\mu_{m}$ approaches infinity at an extremely fast rate. In particular, by defining every element in 
the sequence with dependence on the previous one, we may assume that

$$
\frac{R_{m-1}^{d}}{\mu_{m}} \stackrel{m \rightarrow \infty}{\longrightarrow} 0 .
$$

Using this notation, Theorem 1.1 follows from Lemmas 5.1 and 5.2 below.

LEMMA 5.1. Let $\mathbb{X}$ be a minimal space of Delone sets and assume that there exists $\Lambda \in \mathbb{X}$ that is non-uniformly spread. Let $\left(A_{m}\right)_{m \in \mathbb{N}}$ and $\left(B_{m}\right)_{m \in \mathbb{N}}$ be the sequences of sets in $\mathcal{Q}_{d}^{*}$ defined in Proposition 4.1 and in (5.2), with respect to $\Lambda$. For every word $\omega \in\{A, B\}^{\mathbb{N}}$, let $\left(C_{m}\right)_{m \in \mathbb{N}}$ be the sequence of sets in $\mathcal{Q}_{d}^{*}$ defined by

$$
C_{m} \stackrel{\text { def }}{=} \begin{cases}A_{m}, & \omega(m)=A, \\ B_{m}, & \omega(m)=B,\end{cases}
$$

where $w(m)$ is the mth letter in $w$. Then there exists a sequence $\left(\mathbf{u}_{m}\right)_{m \in \mathbb{N}}$ of vectors in $\mathbb{R}^{d}$ so that $\Lambda_{\omega}=\lim _{m \rightarrow \infty}\left(\Lambda \cap C_{m}\right)-\mathbf{u}_{m}$ is a Delone set in $\mathbb{X}$,

$$
\mathbf{u}_{m} \in \begin{cases}B\left(\mathbf{y}_{m}, R_{m-1}\right), & \omega(m)=A, \\ B\left(\mathbf{p}_{m}, R_{m-1}\right), & \omega(m)=B,\end{cases}
$$

and

$$
\text { for all } m \geq 2, \quad\left|\#\left(\Lambda_{\omega} \cap\left(C_{m}-\mathbf{u}_{m}\right)\right)-\#\left(\Lambda \cap C_{m}\right)\right| \leq c_{3} \cdot \operatorname{vol}_{d-1}\left(\partial C_{m}\right),
$$

where $c_{3}$ is a constant that depends on $d$ and on the separation constant $r(\Lambda)$.

Proof. Given $\omega \in\{A, B\}^{\mathbb{N}}$, consider the sequence $\left(C_{m}\right)_{m \in \mathbb{N}}$ of sets in $\mathcal{Q}_{d}^{*}$ defined by (5.6). From (5.1) and (5.3), conditions (1) and (2) of Proposition 3.3 are being satisfied for $\left(C_{m}\right)_{m \in \mathbb{N}}$, with $\left(\varepsilon_{m}\right)_{m \in \mathbb{N}}$ as described at the beginning of this section. Applying Proposition 3.3, we obtain vectors $\mathbf{u}_{m}$, which satisfies (5.7), for which the sequence of patches $Q_{m} \stackrel{\text { def }}{=}\left(\Lambda \cap C_{m}\right)-\mathbf{u}_{m}$ is convergent. Setting $\Lambda_{\omega}$ to be the limit, by (3.6) of Proposition 3.3 for every $m \geq 2$,

$$
\left|\#\left(\Lambda_{\omega} \cap\left(C_{m}-\mathbf{u}_{m}\right)\right)-\# Q_{m}\right| \leq c_{3} \cdot \varepsilon_{m}^{d} \cdot \operatorname{vol}_{d-1}\left(\partial C_{m}\right),
$$

where $c_{3}$ depends on $d$ and on $r(\Lambda)$. Clearly \# $Q_{m}=\#\left(\Lambda \cap C_{m}\right)$, and (5.8) follows.

LEMMA 5.2. Let $\mathbb{X}$ be a minimal space of Delone sets and assume that there exists $\Lambda \in \mathbb{X}$ that is non-uniformly spread. Let $\eta, \sigma \in\{A, B\}^{\mathbb{N}}$ be two words that differ in infinitely many places. Then, the Delone sets $\Lambda_{\eta}$ and $\Lambda_{\sigma}$ defined in Lemma 5.1 are BD-non-equivalent.

Proof. Taking a subsequence if necessary, we may assume without lose of generality that $\eta$ and $\sigma$ are everywhere different. We use an upper index of $\eta$ or $\sigma$ on elements of $\mathcal{Q}_{d}^{*}$ and on vectors, e.g. $C_{m}^{\eta}$ and $\mathbf{u}_{m}^{\sigma}$, to distinguish between those elements that come from the construction of $\Lambda_{\eta}$ and of $\Lambda_{\sigma}$ in Lemma 5.1.

By denoting $F_{m} \stackrel{\text { def }}{=} C_{m}^{\eta}-\mathbf{u}_{m}^{\eta}$ and using (5.8) for $w=\eta$, we obtain

$$
\text { for all } m \geq 2, \quad\left|\#\left(\Lambda_{\eta} \cap F_{m}\right)-\#\left(\Lambda \cap C_{m}^{\eta}\right)\right| \leq c_{3} \cdot \operatorname{vol}_{d-1}\left(\partial F_{m}\right) .
$$


Observe that for every $m \geq 2$ there exists some $\mathbf{v}_{m} \in \mathbb{R}^{d}$ so that

$$
\left[F_{m}-\mathbf{v}_{m}=\right] \quad\left(C_{m}^{\eta}-\mathbf{u}_{m}^{\eta}\right)-\mathbf{v}_{m}=C_{m}^{\sigma}-\mathbf{u}_{m}^{\sigma} \quad \text { and } \quad\left\|\mathbf{v}_{m}\right\|_{\infty} \leq 2 R_{m-1} .
$$

Indeed, by assuming without loss of generality that $\eta(m)=A$ and $\sigma(m)=B$ and combining (5.2), (5.6), and (5.7) yields that $C_{m}^{\eta}=A_{m}, C_{m}^{\sigma}=A_{m}+\mathbf{x}_{m}, \mathbf{u}_{m}^{\eta} \in B\left(\mathbf{y}_{m}, R_{m-1}\right)$ and $\mathbf{u}_{m}^{\sigma} \in B\left(\mathbf{y}_{m}+\mathbf{x}_{m}, R_{m-1}\right)$, which implies (5.10). It follows that

$$
\text { for all } m \geq 2, \quad\left(C_{m}^{\sigma}-\mathbf{u}_{m}^{\sigma}\right) \triangle F_{m} \subset \partial F_{m}^{\left(+2 R_{m-1}\right)},
$$

and, hence, from (2.1)

$$
\text { for all } m \geq 2, \quad\left|\#\left(\Lambda_{\sigma} \cap F_{m}\right)-\#\left(\Lambda_{\sigma} \cap\left(C_{m}^{\sigma}-\mathbf{u}_{m}^{\sigma}\right)\right)\right| \leq c_{4} \cdot R_{m-1}^{d} \cdot \operatorname{vol}_{d-1}\left(\partial F_{m}\right),
$$

where $c_{4}$ depends on $d$ and on $r(\Lambda)$. Again, from (5.8), this time with $w=\sigma$, we obtain

$$
\text { for all } m \geq 2, \quad\left|\#\left(\Lambda_{\sigma} \cap F_{m}\right)-\#\left(\Lambda \cap C_{m}^{\sigma}\right)\right| \leq\left(c_{3}+c_{4} \cdot R_{m-1}^{d}\right) \operatorname{vol}_{d-1}\left(\partial F_{m}\right) .
$$

Combining this with (5.9), the triangle inequality yields that for every $m \geq 2$,

$$
\begin{aligned}
\left|\#\left(\Lambda_{\eta} \cap F_{m}\right)-\#\left(\Lambda_{\sigma} \cap F_{m}\right)\right| \\
\geq\left|\#\left(\Lambda \cap C_{m}^{\eta}\right)-\#\left(\Lambda \cap C_{m}^{\sigma}\right)\right|-\left|\#\left(\Lambda_{\eta} \cap F_{m}\right)-\#\left(\Lambda \cap C_{m}^{\eta}\right)\right| \\
\quad-\left|\#\left(\Lambda_{\sigma} \cap F_{m}\right)-\#\left(\Lambda \cap C_{m}^{\sigma}\right)\right| \\
\quad \geq\left|\#\left(\Lambda \cap C_{m}^{\eta}\right)-\#\left(\Lambda \cap C_{m}^{\sigma}\right)\right|-c_{5} \cdot R_{m-1}^{d} \cdot \operatorname{vol}_{d-1}\left(\partial F_{m}\right),
\end{aligned}
$$

where $c_{5}$ depends on $d$ and $r(\Lambda)$. Because $C_{m}^{\eta}=A_{m}, C_{m}^{\sigma}=A_{m}+\mathbf{x}_{m}$, and $\operatorname{vol}_{d-1}\left(\partial A_{m}\right)=$ $\operatorname{vol}_{d-1}\left(\partial F_{m}\right)$, combined with (5.4), we have

$$
\left|\#\left(\Lambda_{\eta} \cap F_{m}\right)-\#\left(\Lambda_{\sigma} \cap F_{m}\right)\right| \geq\left(\mu_{m}-c_{5} \cdot R_{m-1}^{d}\right) \operatorname{vol}_{d-1}\left(\partial F_{m}\right),
$$

and together with (5.5), we thus obtain

$$
\frac{\left|\#\left(\Lambda_{\eta} \cap F_{m}\right)-\#\left(\Lambda_{\sigma} \cap F_{m}\right)\right|}{\operatorname{vol}_{d-1}\left(\partial F_{m}\right)} \geq \mu_{m}\left(1-\frac{c_{5} \cdot R_{m-1}^{d}}{\mu_{m}}\right) \stackrel{m \rightarrow \infty}{\longrightarrow} \infty .
$$

Theorem 2.3 then implies that the sets $\Lambda_{\eta}$ and $\Lambda_{\sigma}$ are BD-non-equivalent, as required.

Proof of Theorem 1.1. Let $\mathbb{X}$ be a minimal space of Delone sets. If there exists a uniformly spread $\Lambda \in \mathbb{X}$, then, as noted in $\S 1$, every $\Lambda \in \mathbb{X}$ is uniformly spread, and (1) holds.

Otherwise, there exists some $\Lambda \in \mathbb{X}$ that is non-uniformly spread. Consider the equivalence relation on $\{A, B\}^{\mathbb{N}}$, in which $\eta \sim \sigma$, if $\eta$ and $\sigma$ differ in only finitely many places, and let $\Omega \subset\{A, B\}^{\mathbb{N}}$ be a set of equivalence class representatives. Because every equivalence class in this relation is countable, $|\Omega|=2^{\aleph_{0}}$. For every two distinct words $\eta, \sigma \in \Omega$, Lemma 5.2 implies that $\Lambda_{\eta}$ and $\Lambda_{\sigma}$ are BD-non-equivalent; therefore, $\operatorname{BD}(\mathbb{X}) \geq$ $2^{\aleph_{0}}$. As explained in $\S 1$, the upper bound is trivial and so the proof is complete.

Acknowledgment. We are grateful to the anonymous referee for many insightful suggestions and remarks. 
A. Appendix. $D(\cdot, \cdot)$ is a metric

It is known that the function $D(\cdot, \cdot)$ in $(1.1)$ constitutes a metric on $\mathscr{C}\left(\mathbb{R}^{d}\right)$ when it is capped by $1 / \sqrt{2}$ instead of 1 , see e.g. [LSo, §7]. We show that it is indeed a metric also when capped by 1 . The proof is essentially the same.

Proposition A.1. The function $D(\cdot, \cdot)$ in $(1.1)$ is a metric on $\mathscr{C}\left(\mathbb{R}^{d}\right)$.

Proof. The triangle inequality is the only property that is not immediate. Let $X, Y, Z \in$ $\mathscr{C}\left(\mathbb{R}^{d}\right)$ be three closed sets and let $\varepsilon, \delta>0$ so that

$$
D(X, Y) \leq \varepsilon \quad \text { and } \quad D(Y, Z) \leq \delta .
$$

We must show that $D(X, Z) \leq \varepsilon+\delta$. Clearly this is true if $\varepsilon+\delta \geq 1$, and so we may assume in what follows that $\varepsilon+\delta<1$. We have

$$
X \cap B\left(\mathbf{0}, \frac{1}{\varepsilon+\delta}\right)=X \cap B\left(\mathbf{0}, \frac{1}{\varepsilon}\right) \cap B\left(\mathbf{0}, \frac{1}{\varepsilon+\delta}\right) \stackrel{(\text { A.1) }}{\subset} Y^{(+\varepsilon)} \cap B\left(\mathbf{0}, \frac{1}{\varepsilon+\delta}\right) .
$$

Note that because $\delta<\varepsilon+\delta<1$, the expression

$$
\frac{1}{\delta}-\frac{1}{\varepsilon+\delta}-\varepsilon=\frac{\varepsilon+\delta-\delta-\varepsilon \delta(\varepsilon+\delta)}{\delta(\varepsilon+\delta)}=\frac{\varepsilon(1-\delta(\varepsilon+\delta))}{\delta(\varepsilon+\delta)}
$$

is positive, and so $\varepsilon+(1 /(\varepsilon+\delta))<1 / \delta$. By the triangle inequality, if $B(\mathbf{x}, \varepsilon) \cap$ $B(\mathbf{0},(1 /(\varepsilon+\delta))) \neq \varnothing$ then $\rho(\mathbf{0}, \mathbf{x})<\varepsilon+(1 /(\varepsilon+\delta))<1 / \delta$. Therefore,

$$
Y^{(+\varepsilon)} \cap B\left(\mathbf{0}, \frac{1}{\varepsilon+\delta}\right) \subset\left(Y \cap B\left(\mathbf{0}, \frac{1}{\delta}\right)\right) \stackrel{(+\varepsilon)}{\subset}\left(Z^{(+\delta)}\right)^{(+\varepsilon)} \subset Z^{(+(\varepsilon+\delta))} .
$$

Combining this with (A.2) finishes the proof.

\section{REFERENCES}

[ACG] J. Aliste-Prieto, D. Coronel and J. M. Gambaudo. Linearly repetitive Delone sets are rectifiable. Ann. Inst. H. Poincaré Anal. Non Linéaire 30(2) (2013), 275-290.

[ASW] F. Adiceam, Y. Solomon and B. Weiss. Cut-and-project quasicrystals, lattices, and dense forests. J. Lond. Math. Soc., to appear.

[BG] M. Baake and U. Grimm. Aperiodic Order. Volume 1: A Mathematical Invitation. Cambridge University Press, Cambridge, 2013.

[BK1] D. Burago and B. Kleiner. Separated nets in Euclidean space and Jacobians of biLipschitz maps. Geom. Funct. Anal. 8(2) (1998), 273-282.

[BK2] D. Burago and B. Kleiner. Rectifying separated nets. Geom. Funct. Anal. 12 (2002), 80-92.

[Ch] C. Chabauty. Limite d'ensembles et géométrie des nombres. Bull. Soc. Math. France 78 (1950), 143-151.

[CN] M. I. Cortez and A. Navas. Some examples of repetitive, non-rectifiable Delone sets. Geom. Topol. 20(4) (2016), 1909-1939.

[dH] P. de la Harpe. Spaces of closed subgroups of locally compact groups. Preprint, 2008, arXiv:0807.2030.

[DK] M. Dymond and V. Kaluža. Divergence of separated nets with respect to displacement equivalence. Preprint, 2021, arXiv:2102.13046.

[D01] M. Duneau and C. Oguey. Displacive transformations and quasicrystalline symmetries. J. Phys. 51(1) (1990), 5-19.

[DO2] M. Duneau and C. Oguey. Bounded interpolation between lattices. J. Phys. A 24 (1991), 461-475.

[DSS] W. A. Deuber, M. Simonovits and V. T. Sós. A note on paradoxical metric spaces. Studia Sci. Math. Hungar. 30(1) (1995), 17-24.

[Fe] J. M. Fell. A Hausdorff topology for the closed subsets of a locally compact non-Hausdorff space. Proc. Amer. Math. Soc. 13(3) (1962), 472-476. 
[FG] D. Frettlöh and A. Garber. Pisot substitution sequences, one dimensional cut-and-project sets and bounded diameter sets with fractal boundary. Indag. Math. (N.S.) 29(4) (2018), 1114-1130.

[FGS] D. Frettlöh, A. Garber and L. Sadun. Number of bounded distance equivalence classes in hulls of repetitive Delone sets. Preprint, 2021, arXiv:2101.02514.

[FR] D. Frettlöh and C. Richard. Dynamical properties of almost repetitive Delone sets. Discrete Contin. Dyn. Syst. 34(2) (2014), 531-556.

[FSS] D. Frettlöh, Y. Smilansky and Y. Solomon. Bounded displacement non-equivalence in substitution tilings. J. Combin. Theory Ser. A 177 (2021), 105326.

[Fu] H. Furstenberg. Recurrence in Ergodic Theory and Combinatorial Number Theory. Princeton University Press, Princeton, NJ, 1981.

[HK] A. Haynes and H. Koivusalo. Constructing bounded remainder sets and cut-and-project sets which are bounded distance to lattice. Israel J. Math. 212 (2016), 189-201.

[HKK] A. Haynes, M. Kelly and H. Koivusalo. Constructing bounded remainder sets and cut-and-project sets which are bounded distance to lattice, II. Indag. Math. (N.S.) 28(1) (2017), 138-144.

[HKW] A. Haynes, M. Kelly and B. Weiss. Equivalence relations on separated nets arising from linear toral flows. Proc. Lond. Math. Soc. 109(5) (2014), 1203-1228.

[KL] J. Kellendonk and D. Lenz. Equicontinuous Delone dynamical systems. Canad. J. Math. 65(1) (2013), 149-170.

[KS1] M. Kelly and L. Sadun. Pattern equivariant cohomology and theorems of Kesten and Oren. Bull. Lond. Math. Soc. 47(1) (2015), 13-20.

[KS2] M. Kelly and L. Sadun. Pattern equivariant mass transport in aperiodic tilings and cohomology. Int. Math. Res. Not. IMRN (2020), rnz310, doi: 10.1093/imrn/rnz310.

[La] M. Laczkovich. Uniformly spread discrete sets in $\mathbb{R}^{d}$. J. Lond. Math. Soc. 46(2) (1992), 39-57.

[LP] J. C. Lagarias and P. A. B. Pleasants. Repetitive Delone sets and quasicrystals. Ergod. Th. \& Dynam. Sys. 23 (2003), 831-867.

[LSo] J. Y. Lee and B. Solomyak. On substitution tilings and Delone sets without finite local complexity. Discrete Contin. Dyn. Syst. 39(6) (2019), 3149-3177.

[LSt] D. Lenz and P. Stollmann. Delone dynamical systems and associated random operators. Operator Algebras and Mathematical Physics (Constanta, 2001). Ed. J. M. Combes, J. Cuntz, G. A. Elliott, G. Nenciu, H. Siedentop and S. Stratila. Theta, Bucharest, 2003, pp. 267-285.

[Mag] A. N. Magazinov. The family of bi-Lipschitz classes of Delone sets in Euclidean space has the cardinality of the continuum. Proc. Steklov Inst. Math. 275 (2011), 87-98.

[McM] C. T. McMullen. Lipschitz maps and nets in Euclidean space. Geom. Funct. Anal. 8(2) (1998), $304-314$.

[Ra] R. Rado. Factorization of even graphs. Q. J. Math. 1 (1949), 95-104.

[S1] Y. Solomon. Substitution tilings and separated nets with similarities to the integer lattice. Israel J. Math. 181 (2011), 445-460.

[S2] Y. Solomon. A simple condition for bounded displacement. J. Math. Anal. Appl. 414(1) (2014), 134-148.

[S3] Y. Solomon. Continuously many bounded displacement non-equivalences in substitution tiling spaces. J. Math. Anal. Appl. 492(1) (2020), 124426.

[SS] Y. Smilansky and Y. Solomon. Multiscale substitution tilings. Proc. Lond. Math. Soc. doi: 10.1112/plms.12404. Published online 19 April 2021. 